\title{
Down-regulation of long non-coding RNA SNHG14 protects against acute lung injury induced by lipopolysaccharide through microRNA-34c-3p-dependent inhibition of WISP1
}

Jinyuan Zhu' ${ }^{1}$, Jijia Bai ${ }^{1}$, Shaojin Wang ${ }^{2}$ and Hui Dong ${ }^{3^{*}}$

\begin{abstract}
Background: Accumulating evidence has shown the important roles of long non-coding RNAs (IncRNAs) in acute lung injury (ALI). This study aimed to investigate the potential role of IncRNA small nucleolar RNA host gene 14 (SNHG14) in lipopolysaccharides (LPS)-induced ALI.

Methods: Expression of SNHG14, microRNA-34c-3p (miR-34c-3p) and Wnt1 inducible signaling pathway protein 1 (WISP1) in LPS-exposed mouse alveolar macrophages (MH-S) and lung tissues from mice with LPS-induced ALI was determined by reverse transcription quantitative polymerase chain reaction. The interactions among SNHG14, miR-34c$3 p$ and WISP1 were analyzed by dual-luciferase reporter and RIP assays. Using gain-of-function or loss-of-function approaches, the contents of proinflammatory proteins were determined and $\mathrm{MH}-\mathrm{S}$ cell viability was assessed to evaluate the in vitro functions of SNHG14, miR-34c-3p and WISP1, and wet/dry weight ratio and proinflammatory proteins in lung tissues were determined to assess their in vivo effects.

Results: SNHG14 and WISP1 expression was increased, while miR-34c-3p was decreased in ALI models. SNHG14 bound to miR-34c-3p, resulting in impaired miR-34c-3p-dependent down-regulation of WISP1. Both SNHG14 silencing and miR-34c-3p over-expression reduced the levels of proinflammatory proteins IL-18, IL-1 $\beta$, TNF- $a$ and IL- 6 and inhibited MH-S cell viability. SNHG14 silencing or miR-34c-3p over-expression decreased the wet/dry weight ratio in lung tissues from ALI mice. The reductions induced by SNHG14 silencing or miR-34c-3p over-expression were rescued by WISP1 over-expression.
\end{abstract}

Conclusion: This study demonstrated that IncRNA SNHG14 silencing alleviated inflammation in LPS-induced ALI through miR-34c-3p-mediated inhibition of WISP1. Our findings suggest that IncRNA SNHG14 may serve as a therapeutic target for ALI.

Keywords: Long non-coding RNA SNHG14, MicroRNA-34c-3p, Wnt1 inducible signaling pathway protein 1, Acute lung injury, Lipopolysaccharide

\footnotetext{
* Correspondence: 173401951@qq.com

${ }^{3}$ Center of Research Equipment Management, General Hospital of Ningxia Medical University, No. 804, Shengli South Street, Xingqing District, Yinchuan 750004, Ningxia Hui Autonomous Region, People's Republic of China Full list of author information is available at the end of the article
}

(c) The Author(s). 2019 Open Access This article is distributed under the terms of the Creative Commons Attribution 4.0 International License (http://creativecommons.org/licenses/by/4.0/), which permits unrestricted use, distribution, and reproduction in any medium, provided you give appropriate credit to the original author(s) and the source, provide a link to the Creative Commons license, and indicate if changes were made. The Creative Commons Public Domain Dedication waiver (http://creativecommons.org/publicdomain/zero/1.0/) applies to the data made available in this article, unless otherwise stated. 


\section{Introduction}

Acute lung injury (ALI) is a milder form of acute respiratory distress syndrome (ARDS), caused by numerous conditions, such as pneumonia, sepsis, and trauma [1]. ALI is associated with respiratory failure that leads to significant morbidity and sometimes even death [2]. The diagnosis of ALI relies on clinical and radiographic criteria, which are sometimes non-specific, resulting in inaccurate diagnoses [3]. Therefore, the complete understanding of the pathologies and molecular mechanisms of ALI is of great importance in improving the diagnosis and treatment of patients with ALI.

Lipopolysaccharide (LPS) is located in the outer membrane's outer leaflet of many gram-negative bacteria and regarded as a well-characterized pathogen, which is linked with molecular pattern [4]. In addition, LPS also serves as a mediator of pro-inflammation and displays essential characteristics of microvascular lung injury reproducibly when delivered intratracheally [5]. Alveolar macrophages differ from macrophages that are located between the airway epithelium and blood vessels, indicating that macrophage populations are subjected to further specialization within the lungs; and alveolar macrophages are present in tissue compartments, which further suggests that significant fluctuations of environment can lead to differentiation [6]. Strikingly, macrophages such as $\mathrm{MH}-\mathrm{S}$ cells have been recognized to be fundamental to the inflammatory response induced by ALI in addition to leukocytes and endothelial cells. Several studies have also highlighted that regulating their functions can be used as a potential therapeutic option against ALI [7, 8]. Herein, the current study aims to elucidate the functions of mouse alveolar macrophages (MH-S) exposed to LPS for induction of ALI.

Furthermore, long non-coding RNAs (lncRNAs), a relatively new class of heterogeneous non-coding RNAs, have been found in numerous species [9]. LncRNAs play essential roles in biological processes, and as a result lncRNA dysfunctions or dysregulations are linked to various diseases [10]. In addition, lncRNAs are involved in regulating gene expression in multiple ways, including chromosome remodeling and transcriptional and post-transcriptional processing [11]. Down-regulated lncRNA small nucleolar RNA host gene 14 (SNHG14) was previously demonstrated to inhibit the proliferation of non-small cell lung cancer (NSCLC) by inducing cell arrest and apoptosis [12]. Up-regulation of SNHG14, on the other hand, has been recognized to aggravate inflammation in cerebral ische$\mathrm{mia} /$ reperfusion injury by impairing the miR-136-5pdependent inhibition of ROCK1 [13]. Importantly, bioinformatics analysis available at lncRNABase website revealed that SNHG14 might bind to miR-34c-3p. MiRNAs are pivotal gene expression regulators, and consequently have significant roles in several biological processes, including cell metabolism, proliferation, differentiation, and apoptosis [14]. In LPS-treated periodontal ligament cells, miR-34c-3p were identified as a down-regulated miRNA in periodontitis, a chronic inflammatory disease [15]. Moreover, bioinformatics analysis available at microRNA.org website in the early phases of our investigation revealed the existence of possible binding sites between miR-34c-3p and Wnt1inducible signaling pathway protein-1 (WISP1). WISP1, also known as CNN4, belongs to the CCN family and possess the ability to regulate cell growth, transformation and survival in a tissue-specific manner [16]. Another previous study demonstrated that suppression of the WISP1-integrin $\beta 6$ pathway alleviated ALI in a mice model of sepsis [17]. Based on these previous findings, we therefore hypothesize that there is a high possibility that SNHG14, miR-34c-3p, and WISP1 may interact with each other and are implicated in the pathogenesis and progression of ALI.

\section{Materials and methods \\ Ethics statement}

All experimental protocols in the current study were approved by the Ningxia Medical University General Hospital Scientific Research Ethics Committee. Animal related procedures were performed according to the recommendations in the Guide for the Care and Use of Laboratory Animals (8th Edition, 2011, National Research Council).

\section{ALI cell model in vitro}

MH-S cells (Cell resource Center, Chinese Academy of Medical Sciences \& Peking Union Medical College, Beijing, China) were cultured in Ham's F-12 K Medium containing 1.59/L sodium bicarbonate, $15 \%$ fetal bovine serum and $2 \mathrm{mM} \mathrm{L}$-glutamine in a humidified incubator with $5 \% \mathrm{CO}_{2}$ in air at $37^{\circ} \mathrm{C}$. This culture solution was renewed every $2-3$ days.

Cells were inoculated in a 96-well culture plate at a density of $4 \times 10^{5}$ cells $/ \mathrm{mL}$ in $100 \mu \mathrm{L}$ for $1 \mathrm{~h}$. Next, the $12 \mathrm{~K}$ medium was then added to the control group. Except for the blank group, LPS $(100 \mu \mathrm{g} / \mathrm{mL})$ was added to the remaining groups to establish ALI cell models. Subsequently, the cells were transfected using a Lipofectamine 2000 kit (Invitrogen, Thermo Fisher Scientific, Waltham, MA, USA).

\section{ALI mouse model in vivo}

Mice obtained from the Institute of Cancer Research $(n=$ 80, 18-24 g, Hunan SJA Laboratory Animal Co., Ltd., Hunan, China; SCXK, Xiang, 2009-0004) were maintained under specific pathogen-free conditions. The mice were then randomly assigned into the control $(n=10)$ and 
ALI $(n=70)$ groups and intraperitoneally anesthetized with $3 \%$ amobarbital $(100 \mathrm{mg} / \mathrm{kg})$. Mice were placed in a supine position to prepare for subsequent surgical procedures. A $0.5 \mathrm{~cm}$ longitudinal incision was made at the neck approximately $1-1.5 \mathrm{~cm}$ below the teeth. Subcutaneous tissues were carefully separated to expose the trachea. LPS diluted in $300 \mu \mathrm{L}$ sterile normal saline was injected into the trachea of mice in the ALI group, whereas the mice in the control group received an injection of $300 \mu \mathrm{L}$ sterile normal saline. Six hours after LPS administration, the mice were grouped ( $n=10$ mice/group). MH-S cells $\left(1 \times 10^{6}\right.$ cells, $\left.500 \mu \mathrm{L}\right)$ infected with adenovirus harboring SNHG14-antisense oligonucleotide (SNHG14-ASO), miR34c-3p mimic or over-expressed WISP1 (oe-WISP1) (Zhonghong Boyuan Biological Technology Co., Ltd., Shenzhen, China) were injected into the mice via the caudal vein. After $48 \mathrm{~h}$, the trachea was cannulated, the lungs were rinsed with $1.2 \mathrm{~mL}$ phosphate-buffered saline (PBS) 3 times and the solution was collected. Mice were then euthanized and the degree of lung injury was observed.

\section{Subcellular localization of SNHG14 determined by fluorescence in situ hybridization (FISH)}

Ribo $^{\text {tix }}$ lncRNA FISH Probe Mix (red, C10920, Guangzhou RiboBio Co., Ltd., Guangzhou, Guangdong, China) was employed in the current study. Cells $\left(6 \times 10^{4}\right.$ cells/well $)$ were seeded in a 24-well plate and fixed with $1 \mathrm{~mL}$ of $4 \%$ paraformaldehyde for $10 \mathrm{~min}$. Pre-cooled PBS containing $0.5 \%$ Triton X-100 $(1 \mathrm{~mL})$ was added to each well and allowed to stand at $4{ }^{\circ} \mathrm{C}$ for $5 \mathrm{~min}$. Next, the prehybridization solution $(200 \mu \mathrm{L})$ was added and sealed at $37^{\circ} \mathrm{C}$ for $30 \mathrm{~min}$. After removal of the pre-hybridization solution, the hybridization solution containing antiSNHG14 oligonucleotide probes (Wuhan GeneCreate Biological Engineering Co., Ltd., Wuhan, Hubei, China) was added to each well and allowed to hybridize overnight at $37^{\circ} \mathrm{C}$ in dark conditions. On the following day, the cells were washed with cleansing solution I at $42^{\circ} \mathrm{C}(4 \times$ saline sodium citrate (SSC), $0.1 \%$ Tween-20), cleansing solution II $(2 \times$ SSC $)$, cleansing solution III $(1 \times$ SSC $)$ and $1 \times$ PBS successively. Cells were then stained with 4',6-diamidino2-phenylindole at a ratio of 1: 800 for $10 \mathrm{~min}$ and mounted with nail polish. Finally, the cells were observed and imaged in 5 random fields under a fluorescence microscope (Olympus, Tokyo, Japan).

\section{Dual-luciferase reporter assay}

The lncRNABase (http://starbase.sysu.edu.cn/mirLncRNA. php) was applied to predict the downstream miRNAs that could be regulated by SNHG14. In addition, the target genes of miR-34c-3p were analyzed using the online website, microRNA.org. Based on the predicted binding sites, wild-type (wt) SNHG14 sequence and mutant (mut) sequence, as well as WISP1 mRNA 3 '-untranslated region
(3'-UTR) sequences and mutant sequences were synthesized and introduced into the pmirGLO luciferase vectors (E1330, Promega, Madison, WI, USA) to generate wtSNHG14, mut-SNHG14, wt-WISP1, and mut-WISP1 plasmids (GenePharma Co., Ltd., Shanghai, China). Negative control (NC) mimic or miR-34c-3p mimic was cotransfected with wt-SNHG14 (containing ACACACCAAT CACTA that bound to miR-34c-3p), mut-SNHG14 (in absence of ACACACCAATCACTA that bound to miR34c-3p), wt-WISP1 (containing TCACTA that bound to miR-34c-3p) and mut-WISP1 (in absence of TCACTA that bound to miR-34c-3p) into $293 \mathrm{~T}$ cells for $48 \mathrm{~h}$. Dualluciferase reporter assay kits (D0010, Beijing Solarbio Science \& Technology Co., Ltd., Beijing, China) and GLomax20/20 luminometer (E5311, Shaanxi Zhongmei Biotechnology Co., Ltd., Shaanxi, China) were used to determine the luciferase activity.

\section{RNA binding protein immunoprecipitation (RIP)}

RIP lysis buffer (N653-100 mL, Shanghai Haoran Biological Technology Co., Ltd., Shanghai, China) was added to the MH-S cells and maintained on ice for 5 min to prepare the cell lysate. Magnetic beads $(50 \mu \mathrm{L})$ and RIP wash buffer $(0.5 \mathrm{~mL}, \mathrm{EHJ}$-BVIS08102, Xiamen Huijia Biotechnology Co., Ltd., Xiamen, China) were added to each sample tube. After brief mixing, the tubes were placed on a magnetic separator until the beads were collected. RIP wash buffer $(100 \mu \mathrm{L})$ was added to re-suspend the magnetic beads followed by the addition of $5 \mu \mathrm{g}$ of Argonaute2 (Ago2) antibody (P10502500, Otwo Biotech Inc., Shenzhen, China). Immunoglobulin G (IgG) was added instead of the Ago2 antibody as NC. Beads were then washed twice with $0.5 \mathrm{~mL}$ RIP wash buffer. Next, the RIP Immunoprecipitation buffer $(900 \mu \mathrm{L}, \mathrm{P} 10403138$, Otwo Biotech Inc., Shenzhen, China) was added to the bead-antibody mixture and centrifuged at $14,000 \mathrm{rpm}$ at $4{ }^{\circ} \mathrm{C}$ for $10 \mathrm{~min}$. The supernatant was then collected and transferred into new Eppendorf tubes (LBCT015S, Beijing North TZ-Biotech Develop, Co., Ltd., Beijing, China). The mixture $(1 \mathrm{~mL})$ was incubated overnight at $4{ }^{\circ} \mathrm{C}$ with mild rotation to allow uniform mixing of the contents. Beads were then washed with $0.5 \mathrm{~mL}$ RIP wash buffer for 6 times. Proteinase $\mathrm{K}$ buffer $(150 \mu \mathrm{L})$ was added and incubated at $55^{\circ} \mathrm{C}$ for $30 \mathrm{~min}$ to purify the RNA. Total RNA content was extracted using the Trizol method and subjected to reverse transcription quantitative polymerase chain reaction (RT-qPCR).

\section{Enzyme-linked immunosorbent assay (ELISA)}

Cell concentration was adjusted to $4 \times 10^{5}$ cells $/ \mathrm{mL}$ and seeded $(500 \mu \mathrm{L} /$ well $)$ in a 24 -well plate and allowed to culture for $1 \mathrm{~h}$. LPS was added to each well and cultured for another $24 \mathrm{~h}$. Cells were then centrifuged at 1500 
rpm for $10 \mathrm{~min}$ and the supernatant was collected for ELISA.

Bronchoalveolar lavage fluid from ALI mice was stored at $4{ }^{\circ} \mathrm{C}$ and centrifuged at $3000 \mathrm{rpm}$ for $10 \mathrm{~min}$. The supernatant was then collected and stored at $-20^{\circ} \mathrm{C}$ for determination of cytokines; interleukin (IL)-18, IL-1 $\beta$, tumor necrosis factor (TNF)- $\alpha$ and IL-6 using ELISA kits (Multi Science, Hangzhou, China).

\section{Cell counting kit (CCK)-8 assay}

MH-S cells were seeded in a 96-well plate containing $2 \times 10^{3}$ cells/well after transfection for $24 \mathrm{~h}, 48 \mathrm{~h}$ or $72 \mathrm{~h}$ and cultured for an additional $16 \mathrm{~h}$. The culture medium was then replaced by freshly prepared $100 \mu \mathrm{L}$ culture medium containing 10\% CCK-8 and incubated for $1 \mathrm{~h}$. The absorbance value of each well was analyzed with a microplate reader at a wavelength of $450 \mathrm{~nm}$.

\section{Determination of pulmonary edema}

The left lung that was not subjected to bronchoalveolar lavage was collected by means of thoracotomy. The wet (W) weight was recorded and dry (D) weight was measured, after the lung was baked at $80^{\circ} \mathrm{C}$ for $48 \mathrm{~h}$. The W/D weight ratio was then calculated using the following formula: wet weight/dry weight $\times 100 \%$.

\section{Morphological changes in lung tissues determined by hematoxylin-eosin (HE) staining}

The right side of the lung without bronchoalveolar lavage was fixed with formalin solution and prepared into $5 \mu \mathrm{m}$ sections. Sections were then baked at $60^{\circ} \mathrm{C}$ for $1 \mathrm{~h}$ and dewaxed by xylene. After hydration, the sections were stained by HE (Beijing Solarbio Science \& Technology Co., Ltd., Beijing, China), then dehydrated with gradient alcohol, cleared by xylene and mounted with neutral gum. Lung morphological changes were observed under an optical microscope (XP-330, Shanghai Bingyu Optical Instrument Co., Ltd., Shanghai, China).

\section{Measurement of myeloperoxidase (MPO) activity}

Lung tissue homogenates were mixed with thiobarbituric acid (TBA). The mixture was centrifuged and the absorbance per gram of lung tissue was detected in the supernatant using spectrophotometry to evaluate the MPO activity.

\section{Wright-Giemsa staining}

The extracted bronchoalveolar lavage fluid (BALF) was dispersed into a single cell suspension, and the number of cells was counted under a microscope. Then, $15 \mathrm{~mL}$ of the cell suspension was fixed in methanol for 2-3 min, and stained with diluted Wright-Giemsa staining solution (Shanghai Rongbai Biological Technology Co., Ltd., Shanghai, China) for 15-30 min at room temperature.

\section{RT-qPCR}

Total RNA content was extracted using Trizol reagent kits. Primers used are presented in Table 1 (Takara Bio Inc., Tokyo, Japan). Total RNA was reversely transcribed into complementary DNA (cDNA) using PrimeScript RT reagent kits (RRO36A, Takara Biotechnology Ltd., Dalian, Liaoning, China). A SYBR Premix Ex TaqTM II reagent kit (RR820A, Takara) was applied to perform RT-qPCR with an ABI7500 real-time qPCR system (7500, ABI Company, Oyster Bay, NY, USA). U6 was regarded as the internal reference for miR-34c-3p. Glyceraldehyde-3-phosphate dehydrogenase (GAPDH) was used as an internal reference for SNHG14 and WISP1. Relative quantification was calculated using the $2^{-\triangle \triangle C T}$ method.

\section{Western blot analysis}

Total protein content in tissues or cells was extracted by radio-immunoprecipitation assay lysis buffer containing phenylmethylsulfonyl fluoride. Protein concentration was determined by a bicinchoninic acid kit. Next, the proteins were separated by sodium dodecyl sulfatepolyacrylamide gel electrophoresis and transferred onto a polyvinylidene fluoride membrane. Membranes were then blocked with 5\% skim milk powder for $1 \mathrm{~h}$ at room temperature and incubated with the primary antibodies (Abcam Inc., Cambridge, MA, USA) of rabbit antimouse antibodies to WISP1 (ab178547, dilution ratio of $0.5 \mu \mathrm{g} / \mathrm{mL}$ ), caspase-1 (ab1872, dilution ratio of 1: 1000) and GAPDH (ab9485, dilution ratio of 1: 2500, internal reference) overnight at $4{ }^{\circ} \mathrm{C}$. Membranes were then washed with Tris-buffered saline Tween-20 and further incubated with the horseradish peroxidase-conjugated secondary antibody of goat anti-rabbit IgG (ab97051, dilution ratio of 1: 2000; Abcam Inc., Shanghai, China) for $1 \mathrm{~h}$ at room temperature. Proteins on the membrane

Table 1 Primer sequences for RT-qPCR

\begin{tabular}{ll}
\hline SNHG14 & Sequence (5'-3') \\
miR-34c-3p & R: AGCTGCAAGCTITTGACCC \\
& F: GCCCAATCACTAACCACACGG \\
WISP1 & R: GTGCAGGGTCCGAGGT \\
& F: ACCACCTGTGGCCTAGGTAT \\
U6 & R: CCTGCGAGAGTGAAGTTCGT \\
& F: GTGATCACTCCCTGCCTGAG \\
GAPDH & R: GGACTTCACTGGACCAGACG \\
& F: CCGCATCTTCTTGTGCAGTG \\
& R: CCCAATACGGCCAAATCCGT \\
\hline
\end{tabular}

$R T$ - $q P C R$ Reverse transcription quantitative polymerase chain reaction, SNHG14 Small nucleolar RNA host gene 14, miR MicroRNA, WISP1 Wnt1-inducible signaling pathway protein 1, GAPDH Glyceraldehyde-3-phosphate dehydrogenase, $F$ Forward, $R$ Reverse 
were visualized by enhanced chemiluminescence detection kits (BB-3501, Amersham Pharmacia Biotech, UK) and Bio-Rad image analysis system (Bio-Rad Laboratories, Inc. CA, USA). The protein band intensity was determined using the Quantity One v4.6.2 software. The ratio of gray value of target protein band to that of GAPDH was regarded as the relative protein expression.

\section{Statistical analysis}

Statistical analyses were carried out using the SPSS 21.0 software (IBM Corp., Armonk, NY, USA). Measurement data were expressed as mean \pm standard deviation. Comparisons between two groups were analyzed using the unpaired $t$-test. Cell viability at the 24th $\mathrm{h}, 48$ th $\mathrm{h}$ and $72 \mathrm{nd}$ $\mathrm{h}$ was compared by two-way analysis of variance (ANOVA) with non-repeated measure. Pearson's correlation was applied to analyze the correlation between miR$34 c-3 p$ and lncRNA SNHG14 expression. A value of of $p<0.05$ were considered to be statistically significant.

\section{Results}

LncRNA SNHG14 is highly expressed in LPS-treated cell and mouse ALI models

SNHG14 expression was higher in MH-S cells treated with LPS in comparison with the control cells (Fig. 1a). Similarly, SNHG14 was also found to be higher in lung tissues from mice with LPS-induced ALI versus the control mice (Fig. 1b).

Subcellular localization of SNHG14 in MH-S cells was determined using FISH. SNHG14 was found to be primarily expressed in the cytoplasm (Fig. 1c). Subsequently, SNHG14 expression was effectively reduced in MH-S cells by transfection with SNHG14-ASO (Fig. 1d).

On the other hand, pro-inflammatory cytokines were determined in $\mathrm{MH}-\mathrm{S}$ cell supernatant following SNHG14 knock-down by ELISA. SNHG14 silencing decreased TNF- $\alpha$ and IL- 6 levels (Fig. 1e). In addition, CCK-8 assay demonstrated that SNHG14 silencing also decreased MH-S cell viability (Fig. 1f). Taken together, these results showed that SNHG14 was up-regulated in ALI, while SNHG14 silencing may alleviate the inflammatory response in ALI.

\section{miR-34c-3p expression is reduced in LPS-exposed MH-S cells and its up-regulation inhibits LPS-induced acute inflammatory injury}

Initial online analysis revealed the existence of a binding site between SNHG14 and miR-34c-3p (upper panel, Fig. 2a), and subsequently, a dual-luciferase reporter assay was applied to determine this relationship. Luciferase activity was found to be reduced after co-transfection with wt-SNHG14 and miR-34c-3p mimic as compared to the NC mimic (lower panel, Fig. 2a). However, luciferase activity did not vary in cells co-transfected with mut-SNHG14 and miR-34c$3 p$ mimic. These findings suggested a binding relationship between SNHG14 and miR-34c-3p.

Additionally, decreased miR-34c-3p expression was observed in MH-S cells treated with LPS as well as in mice with LPS-induced ALI (Fig. 2b). Pearson's analysis revealed an inverse relationship between SNHG14 and miR-34c-3p expression, wherein increased SNHG14 resulted in a decrease in the expression of miR-34c-3p (Fig. 2c). Furthermore, SNHG14 silencing significantly increased the expression of miR-34c-3p, while no significant differences were detected upon transfection with mut-SNHG14 (Fig. 2d). Therefore, these results demonstrated that SNHG14 bound to and negatively-regulated the expression of miR-34c-3p.

On the other hand, miR-34c-3p mimic-transfected $\mathrm{MH}-\mathrm{S}$ cells were used to determine the effects of miR$34 c-3 p$ on pro-inflammatory cytokines. As expected, miR-34c-3p expression was elevated after miR-34c-3p mimic transfection (Fig. 2e). In addition, the levels of TNF- $\alpha$ and IL- 6 in MH-S cell supernatant were measured using ELISA. The results revealed that miR-34c$3 p$ mimic significantly increased TNF- $\alpha$ and IL- 6 levels (Fig. 2f). Meanwhile, miR-34c-3p mimic transfection resulted in a decrease in MH-S cell viability (Fig. 2g). Taken together, miR-34c-3p was expressed at low-levels in ALI, and over-expression of miR-34c-3p may help alleviate inflammatory reactions during ALI.

\section{LncRNA SNHG14 binds to miR-34c-3p to up-regulate WISP1 expression}

The binding site between miR-34c-3p and WISP1 was originally detected using website analysis (Left panel, Fig. 3a). This allowed us to further analyze whether miR-34c-3p could target and regulate WISP1 to mediate the inflammatory responses in ALI.

We further conducted a dual-luciferase reporter assay, the results of which showed that miR-34c-3p mimic markedly reduced the luciferase activity of wt-WISP1 $(p<0.05)$, but no effects on that of mut-WISP1 (Fig. 3a), which exceedingly suggests the binding of miR-34c-3p to WISP1. In addition, RIP assay showed that anti-Ago2 group exhibited increased expression of SNHG14 or WISP1 binding to miR-34c-3p in MH-S cells when compared to anti-IgG group (Fig. 3b). Moreover, miR-34c-3p mimic transfection decreased the mRNA and protein expression of WISP1 mRNA (Fig. 3c, Fig. 3d), while overexpression SNHG14 neutralized the effects of miR-34c3p. These findings suggested that SNHG14 and WISP1 could competitively bind to miR-34c-3p. Reducing the expression of SNHG14 led to enhanced binding of miR$34 \mathrm{c}-3 \mathrm{p}$ to WISP1, and in turn decreased the WISP1 expression. 
Down-regulated IncRNA SNHG14 alleviates LPS-induced $A L I$ via miR-34c-3p-mediated WISP1 inhibition in vitro $\mathrm{MH}-\mathrm{S}$ exposed to LPS were co-treated with NC-ASO and oe-NC, SNHG14-ASO and oe-NC, SNHG14-ASO and oe-WISP1, NC mimic and oe-NC, miR-34c-3p mimic and oe-NC or miR-34c-3p mimic and oeWISP1. WISP1 mRNA expression was increased in response to SNHG14-ASO + oe-WISP1 treatment when compared with SNHG14-ASO and oe-NC treatment. In addition, WISP1 mRNA expression was increased upon miR-34c-3p mimic and oe-WISP1 treatment than those subjected to miR-34c-3p mimic and oe-NC treatment (Fig. 4a).

According to the results of ELISA, TNF- $\alpha$ and IL-6 levels in the $\mathrm{MH}-\mathrm{S}$ cell supernatant inhibited by either SNHG14-ASO or miR-34c-3p mimic were rescued by oe-WISP1 transfection (Fig. 4b).

In addition, cell viability was measured using CCK-8 assay. The results revealed that the viability of cells cotransfected with SNHG14-ASO and oe-WISP1 was significantly higher compared to that of cells transfected with SNHG14-ASO and oe-NC (Fig. 4c). Cell viability was also increased in response to co-transfection with miR-34c-3p mimic and oe-WISP1 when compared with that of cells co-transfected with miR-34c-3p mimic and oe-NC (Fig. 4c).
Protein expression of WISP1 and caspase-1 was increased in response to SNHG14-ASO and oe-WISP1 cotransfection versus SNHG14-ASO and oe-NC cotransfection. Similarly, miR-34c-3p mimic and oe-WISP1 co-transfection led to increased WISP and caspase-1 protein expression when compared to miR-34c-3p mimic and oe-NC co-transfection (Fig. 4d). Taken together, these results demonstrated that SNHG14 bound to miR-34c-3p to up-regulate WISP1. On the other hand, SNHG14 silencing increased the binding of miR-34c-3p to WISP1 and inhibited WISP1, leading to reduced inflammation in ALI in vitro.

\section{Down-regulated IncRNA SNHG14 alleviates LPS-induced ALI via miR-34c-3p-mediated WISP1 inhibition in vivo}

Microscopic observation revealed that the alveolar structure in the lung from normal mice was intact without thickening or lymphocyte infiltration (left panel, Fig. 5a). However, pulmonary lesions in LPS-treated mice were evident, which presented with pathologically thickened alveolar walls, collapsed alveoli and numerous infiltrated red blood cells and inflammatory cells (right panel, Fig. 5a), indicating that our ALI mice models were successfully established.

Levels of inflammatory factors, IL-18, IL-1, TNF- $\alpha$ and IL-6 in bronchoalveolar lavage fluid were determined to

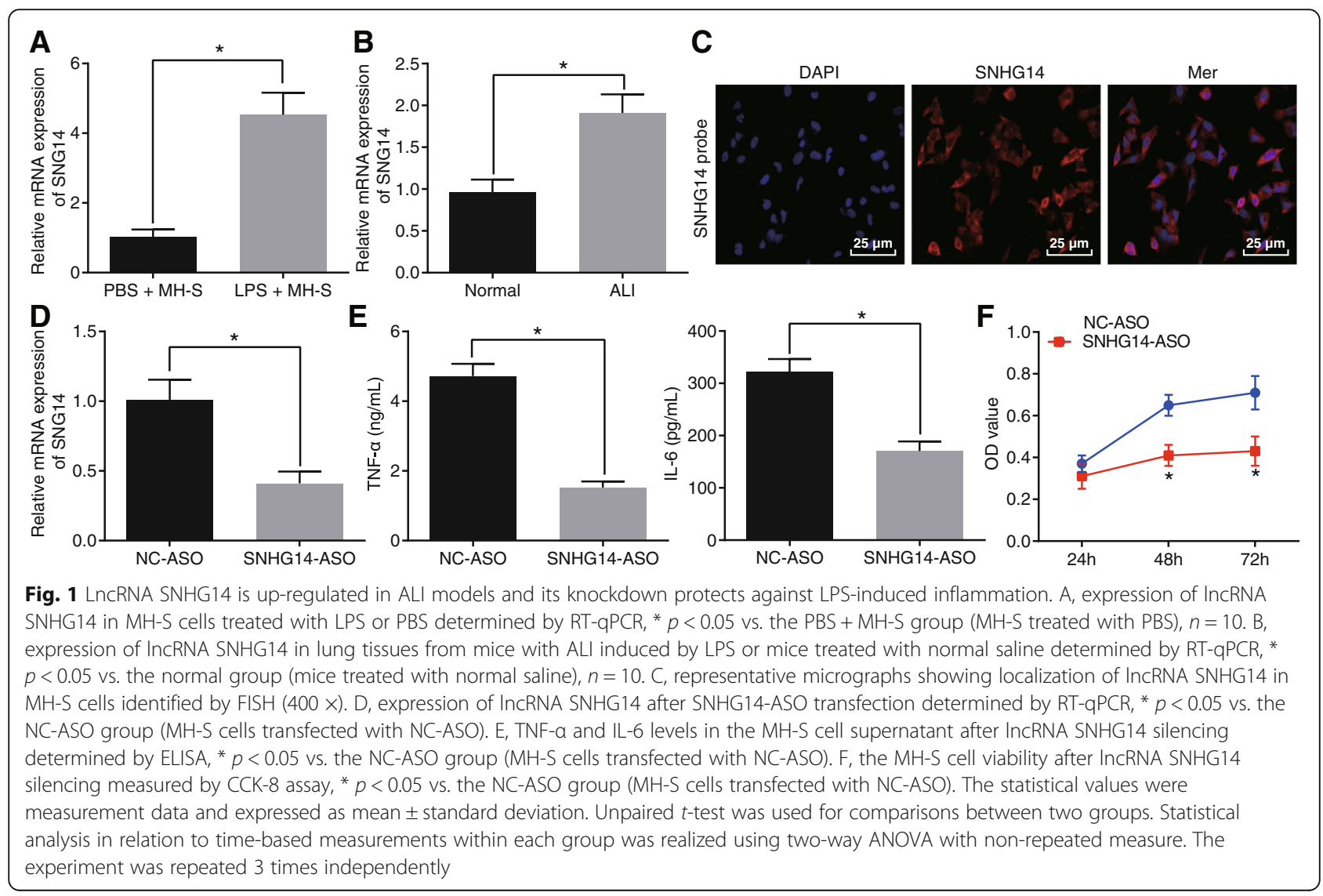




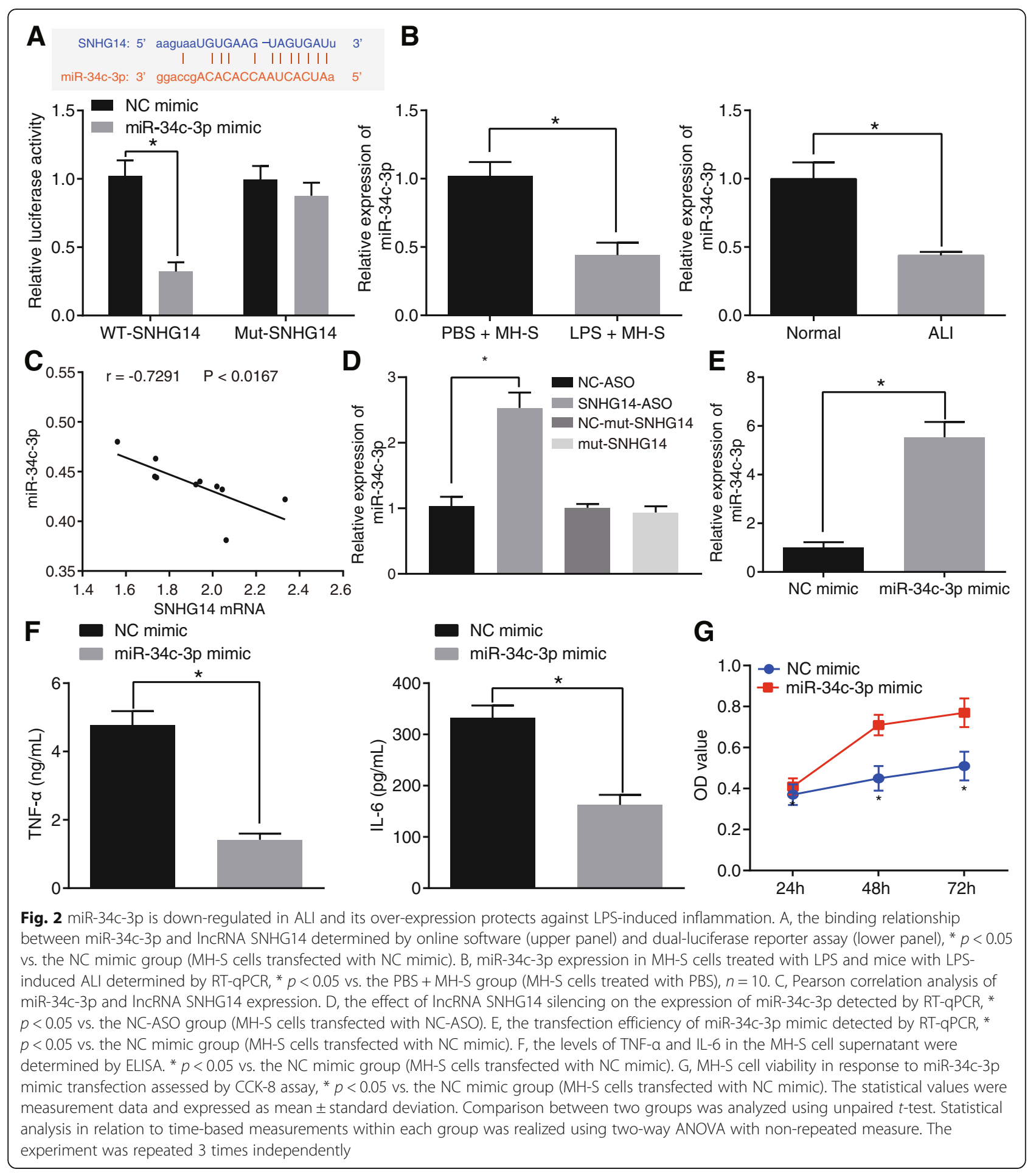

be significantly higher in LPS-treated mice injected with adenoviruses harboring SNHG14-ASO and oe-WISP1 as compared to those in LPS-treated mice injected with adenoviruses harboring SNHG14-ASO and oe-NC (Fig. 5b). In addition, LPS-treated mice injected with miR-34c-3p mimic and oe-WISP1 exhibited markedly increased levels of these inflammatory cytokines when compared to those injected with miR-34c-3p mimic and oe-NC. As shown in Fig. 5c-d after HE staining, aggravated lung tissue damage, alveolar wall thickening, increased alveolar collapse, red blood cell and inflammatory cell infiltration as well as higher lung injury score were observed in LPS-treated mice injected with adenoviruses harboring SNHG14-ASO and oe-WISP1 as compared to those in 


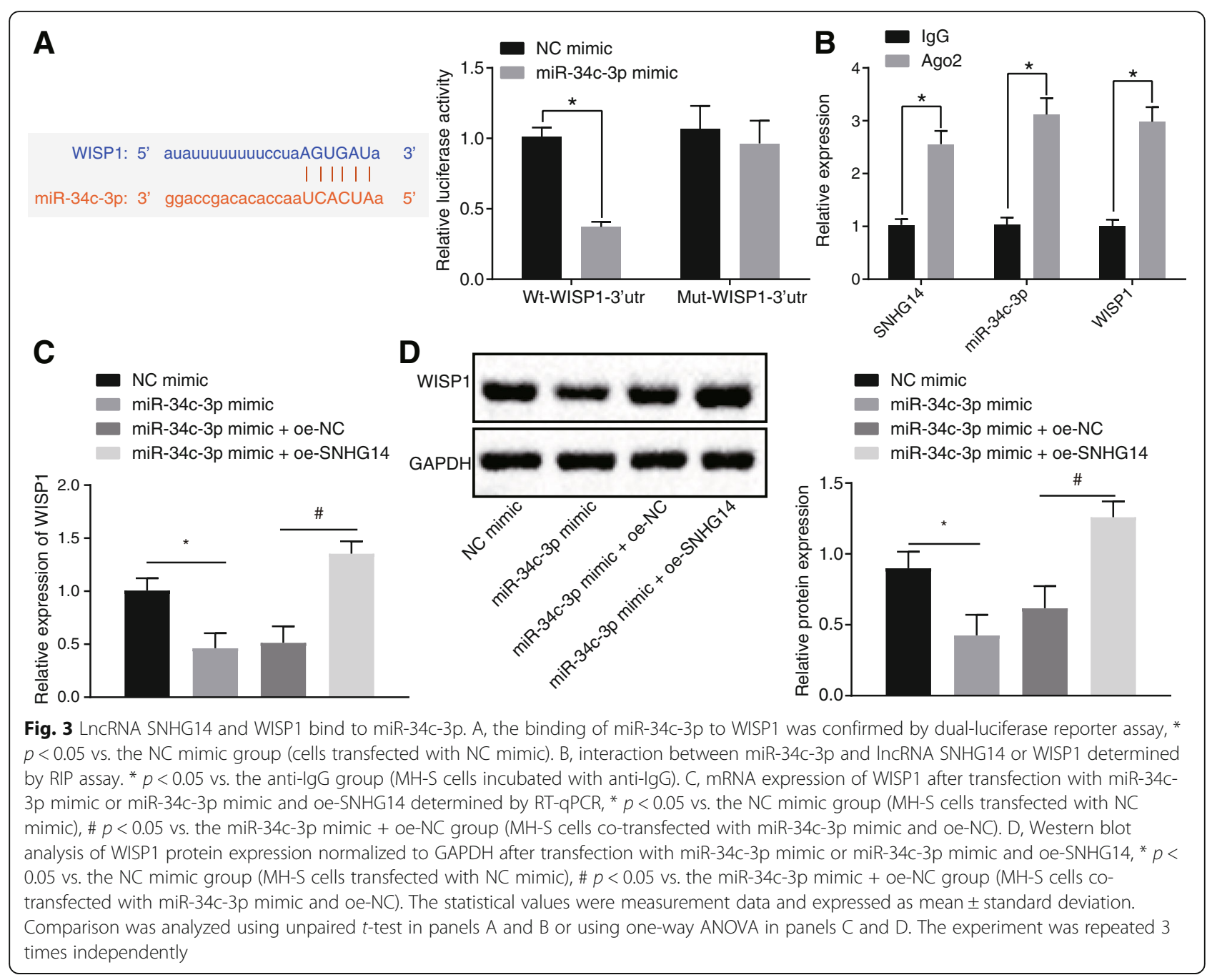

LPS-treated mice injected with adenoviruses harboring SNHG14-ASO and oe-NC. The above-mentioned features of aggravated lung tissue damage were also observed in LPS-treated mice when miR-34c-3p and WISP1 were simultaneously up-regulated as compared to those in LPS-treated mice when only miR-34c-3p was upregulated. Next, measurement of MPO activity and Wright-Giemsa staining were employed to evaluate neutrophil infiltration in lung tissues and BALF. The data displayed that reduced MPO activity and neutrophil infiltration caused by either silencing of SNHG14 or upregulation of miR-34c-3p were rescued by overexpression of WISP1 (Fig. 5e-f). The W/D weight ratio (edema formation) was also calculated to be increased in LPS-treated mice injected with adenoviruses harboring SNHG14-ASO and oe-WISP1 when compared with LPS-treated mice injected with adenoviruses harboring SNHG14-ASO and oe-NC. Similarly, LPS-treated mice injected with adenoviruses harboring miR-34c-3p mimic and oe-WISP1 exhibited increased W/D weight ratio when compared with LPS-treated mice injected with adenoviruses harboring miR-34c-3p mimic and oe-NC (Fig. 5g). These findings suggested that SNHG14 was able to bind to miR-34c-3p and thus, up-regulate WISP1. SNHG14 silencing increased the binding of miR-34c-3p to WISP1 and down-regulated WISP1, leading to reduced inflammation in ALI in vivo.

\section{Discussion}

ALI is defined as a syndrome of acute inflammation and increased permeability in the alveolar-capillary membrane associated with at least one risk factor [18]. Notably, miRNAs have been indicated to play a key role in the regulation of genes implicated in the process of acute inflammatory lung injury [19]. In this study, we found that miR-34c-3p was targeted and down-regulated by lncRNA SNHG14, leading to increased WISP1 in both cell and mice models of LPS-induced ALI. On the contrary, lncRNA SNHG14 silencing resulted in an upregulation of miR-34c-3p and subsequent down- 


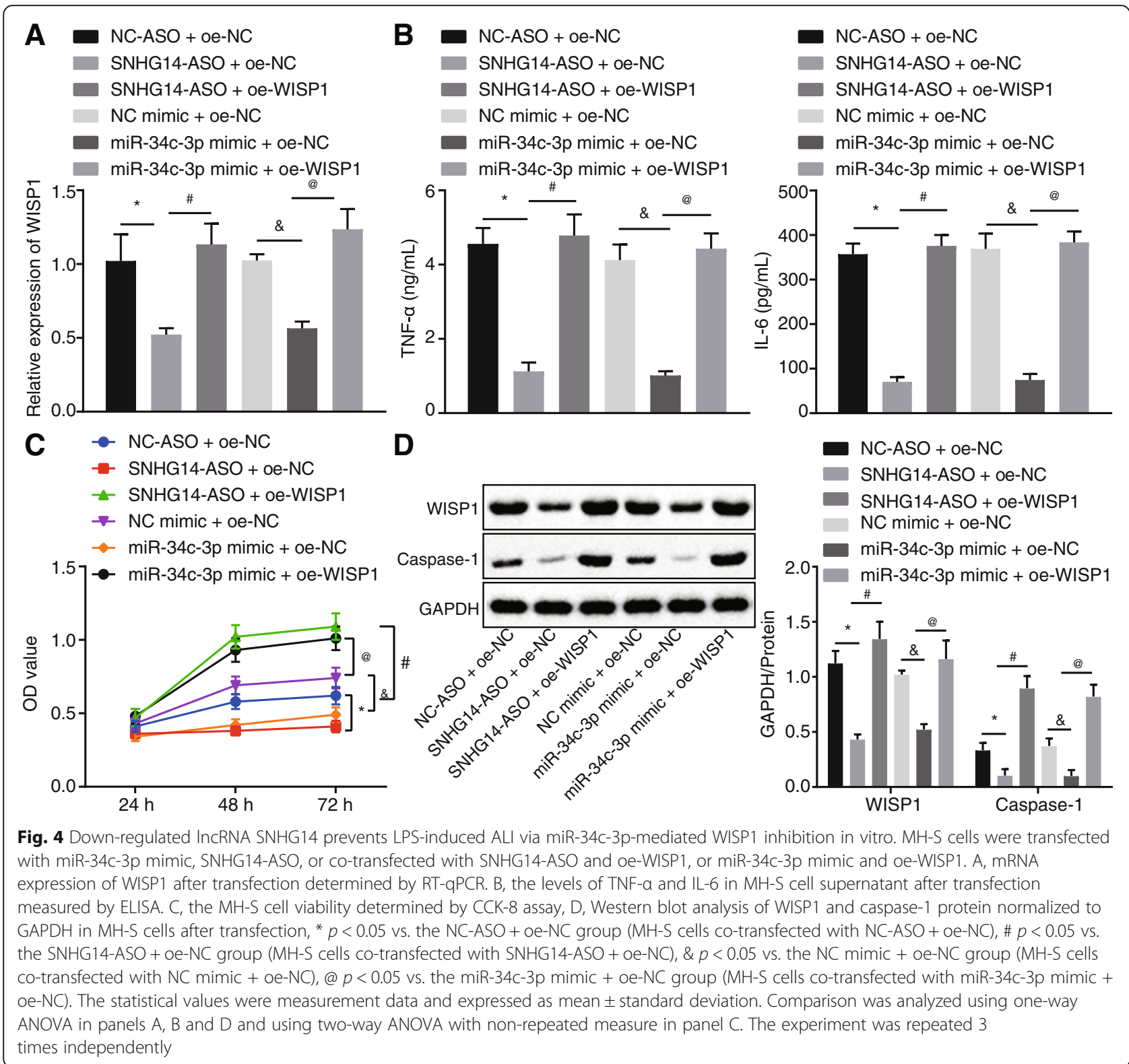

regulation of WISP1 expression, leading to reduced ALI severity.

Initially, we uncovered that LPS-exposed MH-S cells and LPS-injured lung tissues of mice exhibit high expression of lncRNA SNHG14. Similarly, up-regulated levels of SNHG14 have also been documented in oxygen-lacking conditions such as in cerebral ischemic animals and/or oxygen-glucose deprived cells [20]. Moreover, another study has reported that elevated expression of IncRNA SNHG14 in ischemic brain tissues contributes to inflammatory response and neurological impairment, which is partially in line with our findings [13]. Our results and findings suggest the use of SNHG14 silencing to prevent LPS-induced inflammation, thereby protecting against ALI. Previous studies have also echoed our sentiment statement that downregulation of lncRNA MALAT1 suppresses the inflammatory response in ALI rats [21]. Additionally, SNHG14 knockdown suppresses the activation of microglia cells which release inflammatory cytokines following ischemic stroke, in so doing conferring protection against cerebral infarction [22]. Our results also indicate towards a similar regulation, wherein SNHG14 silencing led to a decrease in the viability of MH-S cells and reduction in the release of inflammatory proteins. Another significant finding was that the expression of miR-34c-3p was reduced in LPS-induced ALI tissues. Moreover, overexpression of miR-34c-3p was previously demonstrated to inhibit the progression of LPS-induced ALI. Available data also suggests that miRNAs are involved in 


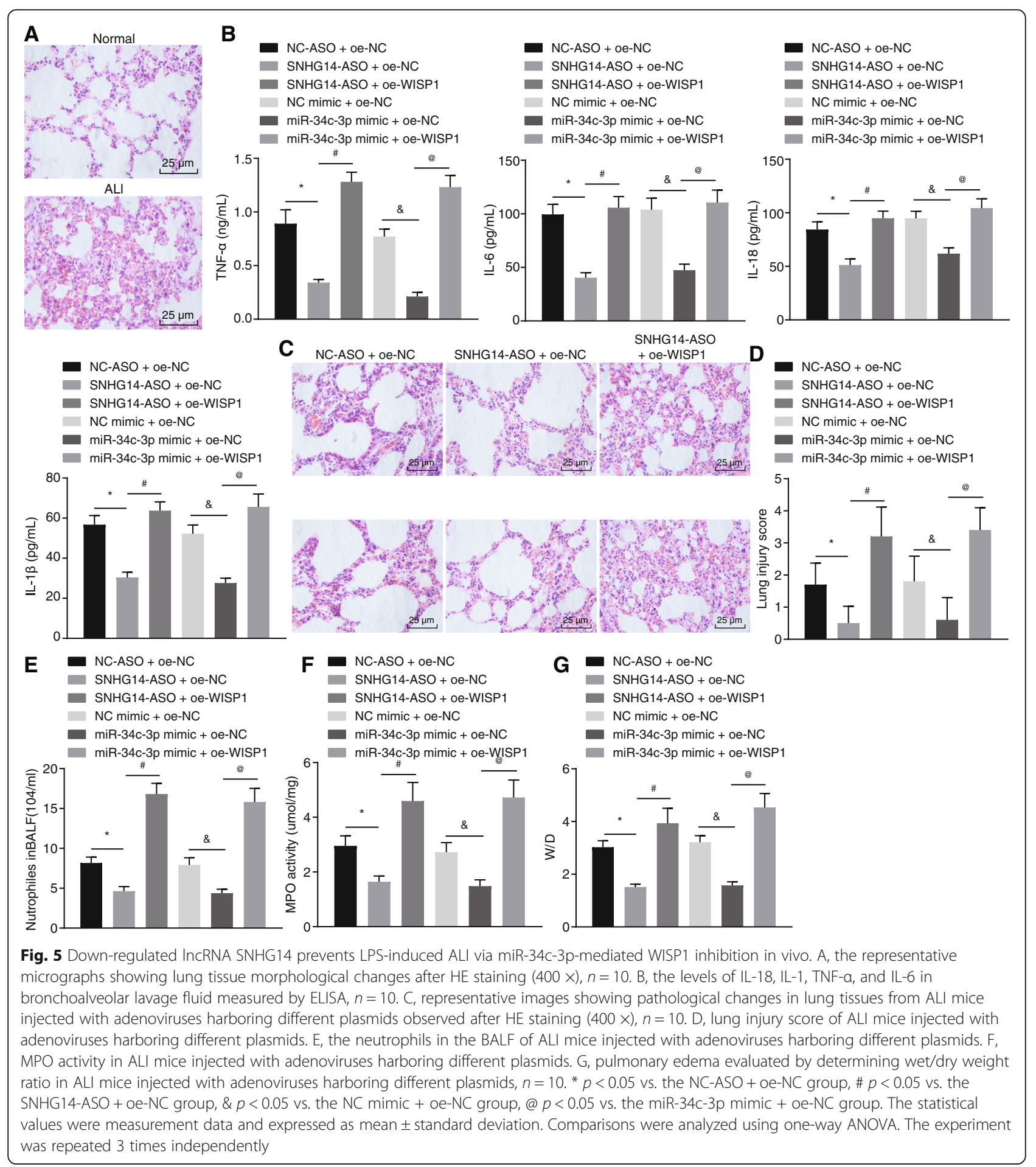

polarization of macrophages bearing critical roles in ALI/ARDS pathogenesis [23]. MiR-34c-3p also exhibits down-regulated expression in the lung tissues of silicaexposed rats, which may be involved in the process of pulmonary fibrosis in early silicosis [24]. Our study evidenced that miR-34c-3p suppresses LPS-induce inflammation and viability of MH-S cells to alleviate ALI.
Given the above-mentioned findings, the involvement of lncRNA SNHG14 and miR-34c-3p in the pathogenesis of ALI is validated.

Additionally, mechanistic analyses demonstrated that lncRNA SNHG14 upregulates the WISP1 gene via binding to miR-34c-3p. WISP1 is not only expressed in multiple different tissues [16], but also up-regulated in 


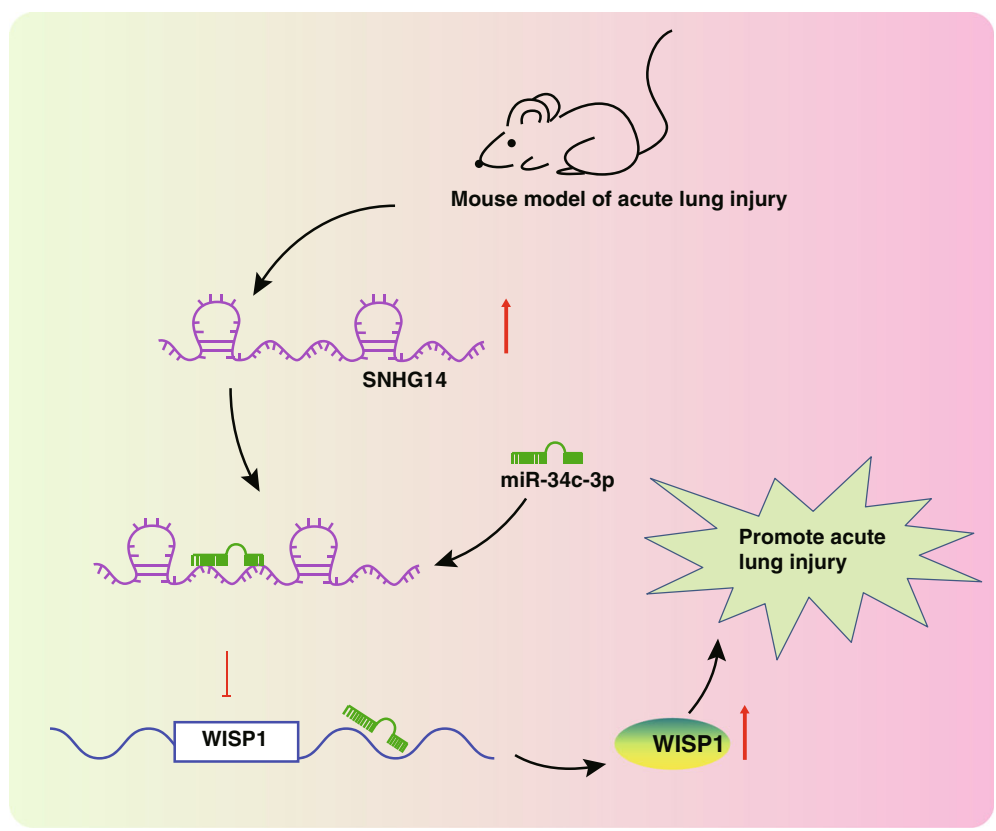

Fig. 6 The schematic diagram showing the mechanisms responsible for the promotive role of IncRNA SNHG14 in ALI. LnCRNA SNHG14 binds to miR-34c-3p to up-regulate WISP1. SNHG14 aggravates LPS-induced ALI via impairing miR-34c-3p-mediated WISP1 down-regulation

ventilator-induced lung injury models [25]. Moreover, recent reports have indicated that WISP1 is regulated by other miRNAs. For instance, miR-92a targets and negatively-regulates WISP1, which prevents the development of idiopathic pulmonary fibrosis [26]. Similar regulatory mechanisms have also been identified, wherein lncRNA SNHG14 binds to miR-193a-3p in breast cancer, and silencing of lncRNA SNHG14 up-regulates miR-193a-3p to inhibit the growth and invasion of breast cancer cells [27]. Besides, lncRNA SNHG14 also downregulates miR-101 to facilitate cell progression in pancreatic ductal adenocarcinoma, while we discovered that lncRNA SNHG14 performs a likewise role in ALI [28]. Furthermore, our findings established that both lncRNA SNHG14 silencing or miR-34c-3p over-expression are capable of reducing the levels of pro-inflammatory cytokines (IL-18, IL-1, TNF- $\alpha$ and IL-6), and thereby alleviate pulmonary edema in ALI models via down-regulation of WISP1. A previous research has already illustrated that IL-18 as well as caspase-1 plays critical roles in the development of lung injury like ARDS [29]. In addition, another study demonstrated that LPS-induced ALI rats express significantly up-regulated levels of pro-inflammatory cytokines TNF- $\alpha$, IL- 6 , and IL-1 $\beta$ during lung injury [30]. Also, a number of miRNAs have been explained to be regulated dynamically in LPS induced ALI mice, among which miR-16 was significantly downregulated, and overexpression of miR-16 led to a reduction in IL-6 and TNF$\alpha$ expression [31]. The promoting effects of WISP1 on the release of TNF- $\alpha$ in macrophages have also been documented in sepsis-induced lung injury, suggesting it functions as a contributor of inflammation [32]. Largely in agreement with our findings, over-expressed miR-145-5p has been revealed to diminish the high levels of TNF- $\alpha$ in BV-2 cells induced by over-expressed SNHG14 [22]. These results provide evidence supporting the regulatory mechanism of the lncRNA SNHG14/miR-34c-3p/WISP1 axis in ALI.

\section{Conclusion}

Returning to the initial hypothesis posed at the beginning of this study, it would be reasonable to state that lncRNA SNHG14 knockdown confers protection against ALI via miR-34c-3p-mediated down-regulation of WISP1. After lncRNA SNHG14 is down-regulated, the binding of miR-34c-3p to WISP1 is increased, leading to depletion of WISP1, thus alleviating ALI severity (Fig. 6). Therefore, lncRNA SNHG14 may serve as a novel therapeutic approach to ALI. However, genetic mismatch effects of alveolar macrophage cell line derived from $\mathrm{BALB} / \mathrm{c}$ mice (MH-S cells) and ICR mice might limit the generalization of our findings. Nevertheless, the current study provides innovative perspectives to advance the hepatoprotective therapy. Further efforts are warranted to validate the generalization for effective clinical applications. Moreover, the interaction between extra-cellular terminally differentiated cells on endothelium has not been studied yet due to current limited conditions. This would be another interesting topic for future researches. 


\section{Abbreviations}

ALI: Acute lung injury; ARDS: Acute respiratory distress syndrome; LPS: Lipopolysaccharides; NSCLC: Non-small cell lung cancer; WISP1: Wnt1inducible signaling pathway protein-1

\section{Acknowledgements}

We would like to give our sincere appreciation to the reviewers for their helpful comments on this article.

\section{Authors' contributions}

$J Z, J B, S W$ and HD designed the study. JZ collated the data, carried out data analyses and produced the initial draft of the manuscript. JB, SW and HD contributed to drafting the manuscript. All authors have read and approved the final submitted manuscript.

\section{Funding}

This work was supported by the NingXia Natural Science Foundation of China (No. 2019AAC03182) and the Plan of Talented Personnel Project for Youth in Ningxia Autonomous Region (2017). The funders had no role in study design, data collection and analysis, decision to publish, or preparation of the manuscript.

\section{Availability of data and materials}

The datasets generated/analysed during the current study are available.

\section{Ethics approval and consent to participate}

All experimental protocols in the current study were approved by the Ningxia Medical University General Hospital Scientific Research Ethics Committee. Animal related procedures were performed according to the recommendations in the Guide for the Care and Use of Laboratory Animals (8th Edition, 2011, National Research Council).

\section{Consent for publication}

Not applicable.

\section{Competing interests}

The authors declare that they have no competing interests.

\section{Author details}

'Department of Critical Care Medicine, General Hospital of Ningxia Medical University, Yinchuan 750004, People's Republic of China. ${ }^{2}$ Department of Respiratory and Critical Care Medicine, General Hospital of Ningxia Medical University, Yinchuan 750004, People's Republic of China. ${ }^{3}$ Center of Research Equipment Management, General Hospital of Ningxia Medical University, No. 804, Shengli South Street, Xingqing District, Yinchuan 750004, Ningxia Hui Autonomous Region, People's Republic of China.

\section{Received: 5 June 2019 Accepted: 9 October 2019}

\section{Published online: 28 October 2019}

\section{References}

1. Mokra D, Kosutova P. Biomarkers in acute lung injury. Respir Physiol Neurobiol. 2015;209:52-8.

2. Butt $Y$, Kurdowska A, Allen TC. Acute lung injury: a clinical and molecular review. Arch Pathol Lab Med. 2016;140:345-50.

3. Elicker BM, Jones KT, Naeger DM, Frank JA. Imaging of Acute Lung Injury. Radiol Clin N Am. 2016;54:1119-32.

4. Park BS, Lee JO. Recognition of lipopolysaccharide pattern by TLR4 complexes. Exp Mol Med. 2013;45:e66.

5. Mirzapoiazova T, Kolosova IA, Moreno L, Sammani S, Garcia JG, Verin AD. Suppression of endotoxin-induced inflammation by taxol. Eur Respir J. 2007; 30:429-35.

6. Hussell T, Bell TJ. Alveolar macrophages: plasticity in a tissue-specific context. Nat Rev Immunol. 2014;14:81-93.

7. Huang X, Xiu H, Zhang S, Zhang G. The role of macrophages in the pathogenesis of ALI/ARDS. Mediat Inflamm. 2018;2018:1264913.

8. Yang L, Zhang Z, Zhuo Y, Cui L, Li C, Li D, Zhang S, Cui N, Wang X, Gao H. Resveratrol alleviates sepsis-induced acute lung injury by suppressing inflammation and apoptosis of alveolar macrophage cells. Am J Transl Res. 2018;10:1961-75.
9. Fatica A, Bozzoni I. Long non-coding RNAs: new players in cell differentiation and development. Nat Rev Genet. 2014;15:7-21.

10. Chen G, Wang Z, Wang D, Qiu C, Liu M, Chen X, Zhang Q, Yan G, Cui Q. LncRNADisease: a database for long-non-coding RNA-associated diseases. Nucleic Acids Res. 2013;41:D983-6.

11. Shi X, Sun M, Liu H, Yao Y, Song Y. Long non-coding RNAs: a new frontier in the study of human diseases. Cancer Lett. 2013;339:159-66.

12. Zhang Z, Wang Y, Zhang W, Li J, Liu W, Lu W. Long non-coding RNA SNHG14 exerts oncogenic functions in non-small cell lung cancer through acting as an miR-340 sponge. Biosci Rep. 2019;39:BSR20180941.

13. Zhong Y, Yu C, Qin W. LncRNA SNHG14 promotes inflammatory response induced by cerebral ischemia/reperfusion injury through regulating miR136-5p /ROCK1. Cancer Gene Ther. 2019;26:234-47.

14. Huntzinger $E$, Izaurralde $E$. Gene silencing by microRNAs: contributions of translational repression and mRNA decay. Nat Rev Genet. 2011;12: 99-110.

15. Du A, Zhao S, Wan L, Liu T, Peng Z, Zhou Z, Liao Z, Fang H. MicroRNA expression profile of human periodontal ligament cells under the influence of Porphyromonas gingivalis LPS. J Cell Mol Med. 2016;20: 1329-38.

16. Ferrand N, Bereziat V, Moldes M, Zaoui M, Larsen AK, Sabbah M. WISP1/ CCN4 inhibits adipocyte differentiation through repression of PPARgamma activity. Sci Rep. 2017:7:1749.

17. Ding X, Wang X, Zhao X, Jin S, Tong Y, Ren H, Chen Z, Li Q. RGD peptides protects against acute lung injury in septic mice through Wisp1-integrin beta6 pathway inhibition. Shock. 2015;43:352-60.

18. Costa EL, Amato MB. The new definition for acute lung injury and acute respiratory distress syndrome: is there room for improvement? Curr Opin Crit Care. 2013;19:16-23.

19. Rao R, Nagarkatti $P$, Nagarkatti $M$. Role of miRNA in the regulation of inflammatory genes in staphylococcal enterotoxin B-induced acute inflammatory lung injury and mortality. Toxicol Sci. 2015;144:284-97.

20. Bao MH, Szeto V, Yang BB, Zhu SZ, Sun HS, Feng ZP. Long non-coding RNAs in ischemic stroke. Cell Death Dis. 2018;9:281.

21. Dai L, Zhang G, Cheng Z, Wang X, Jia L, Jing X, Wang H, Zhang R, Liu M, Jiang $T$, et al. Knockdown of LncRNA MALAT1 contributes to the suppression of inflammatory responses by up-regulating miR-146a in LPSinduced acute lung injury. Connect Tissue Res. 2018;59:581-92.

22. Qi X, Shao M, Sun H, Shen Y, Meng D, Huo W. Long non-coding RNA SNHG14 promotes microglia activation by regulating miR-145-5p/PLA2G4A in cerebral infarction. Neuroscience. 2017;348:98-106.

23. Rajasekaran S, Pattarayan D, Rajaguru P, Sudhakar Gandhi PS, Thimmulappa RK. MicroRNA regulation of acute lung injury and acute respiratory distress syndrome. J Cell Physiol. 2016;231:2097-106.

24. Wang JY, Geng X, Jia Q, Li C, Sai LL, Yu GC, Shao H. Expression changes of miRNA-29b-3p and miRNA-34c-3p in lung tissue of rats exposed to silica and A549 cells. Zhonghua Lao Dong Wei Sheng Zhi Ye Bing Za Zhi. 2019; 37:110-5.

25. Li HH, Li Q, Liu P, Liu Y, Li J, Wasserloos K, Chao W, You M, Oury TD, Chhinder $\mathrm{S}$, et al. WNT1-inducible signaling pathway protein 1 contributes to ventilator-induced lung injury. Am J Respir Cell Mol Biol. 2012;47:528-35.

26. Berschneider B, Ellwanger DC, Baarsma HA, Thiel C, Shimbori C, White ES, Kolb M, Neth P, Konigshoff M. miR-92a regulates TGF-beta1-induced WISP1 expression in pulmonary fibrosis. Int J Biochem Cell Biol. 2014;53:432-41

27. Xie SD, Qin C, Jin LD, Wang QC, Shen J, Zhou JC, Chen YX, Huang AH, Zhao WH, Wang LB. Long noncoding RNA SNHG14 promotes breast cancer cell proliferation and invasion via sponging miR-193a-3p. Eur Rev Med Pharmacol Sci. 2019;23:2461-8.

28. Zhang $X$, Zhao P, Wang C, Xin B. SNHG14 enhances gemcitabine resistance by sponging miR-101 to stimulate cell autophagy in pancreatic cancer. Biochem Biophys Res Commun. 2019;510:508-14.

29. Dolinay T, Kim YS, Howrylak J, Hunninghake GM, An CH, Fredenburgh L, Massaro AF, Rogers A, Gazourian L, Nakahira K, et al. Inflammasomeregulated cytokines are critical mediators of acute lung injury. Am J Respir Crit Care Med. 2012;185:1225-34.

30. Zeng Z, Gong H, Li Y, Jie K, Ding C, Shao Q, Liu F, Zhan Y, Nie C, Zhu W, Qian K. Upregulation of miR-146a contributes to the suppression of inflammatory responses in LPS-induced acute lung injury. Exp Lung Res. 2013;39:275-82. 
31. Cai ZG, Zhang SM, Zhang Y, Zhou YY, Wu HB, Xu XP. MicroRNAs are dynamically regulated and play an important role in LPS-induced lung injury. Can J Physiol Pharmacol. 2012;90:37-43.

32. Chen Z, Ding X, Jin S, Pitt B, Zhang L, Billiar T, Li Q. WISP1-alphavbeta3 integrin signaling positively regulates TLR-triggered inflammation response in sepsis induced lung injury. Sci Rep. 2016;6:28841.

\section{Publisher's Note}

Springer Nature remains neutral with regard to jurisdictional claims in published maps and institutional affiliations.

Ready to submit your research? Choose BMC and benefit from:

- fast, convenient online submission

- thorough peer review by experienced researchers in your field

- rapid publication on acceptance

- support for research data, including large and complex data types

- gold Open Access which fosters wider collaboration and increased citations

- maximum visibility for your research: over $100 \mathrm{M}$ website views per year

At $\mathrm{BMC}$, research is always in progress.

Learn more biomedcentral.com/submissions 